\title{
PDEs satisfied by extreme eigenvalues distributions of GUE and LUE
}

\author{
Estelle Basor*, Yang Chen†, Lun Zhang
}

May 8, 2022

\begin{abstract}
In this paper we study, $\operatorname{Prob}(n, a, b)$, the probability that all the eigenvalues of finite $n$ unitary ensembles lie in the interval $(a, b)$. This is identical to the probability that the largest eigenvalue is less than $b$ and the smallest eigenvalue is greater than $a$. It is shown that a quantity allied to $\operatorname{Prob}(n, a, b)$, namely,

$$
H_{n}(a, b):=\left[\frac{\partial}{\partial a}+\frac{\partial}{\partial b}\right] \ln \operatorname{Prob}(n, a, b),
$$

in the Gaussian Unitary Ensemble (GUE) and

$$
H_{n}(a, b):=\left[a \frac{\partial}{\partial a}+b \frac{\partial}{\partial b}\right] \ln \operatorname{Prob}(n, a, b),
$$

in the Laguerre Unitary Ensemble (LUE) satisfy certain nonlinear partial differential equations for fixed $n$, interpreting $H_{n}(a, b)$ as a function of $a$ and $b$. These partial differential equations maybe considered as two variable generalizations of a Painlevé IV and a Painlevé V system, respectively. As an application of our result, we give an analytic proof that the extreme eigenvalues of the GUE and the LUE, when suitably centered and scaled, are asymptotically independent.
\end{abstract}

*American Institute of Mathematics, Palo Alto, California 94306, USA, E-mail address: ebasor@aimath.org.

${ }^{\dagger}$ Department of Mathematics, Imperial College London, 180 Queen's Gate, London SW7 2BZ, UK. E-mail address: y.chen@imperial.ac.uk.

${ }^{\ddagger}$ Department of Mathematics, Katholieke Universiteit Leuven, Celestijnenlaan 200B, B-3001 Leuven, Belgium. E-mail address: lun.zhang@wis.kuleuven.be. 


\section{Introduction}

In the theory of random matrices, the study of eigenvalue distribution attracts the most interest and has many applications in both mathematic, physics and wireless communications; see for example [3, 18, 21, 27]. It is by now a classic result that the largest eigenvalue distribution of the Gaussian Unitary Ensemble (GUE) and Laguerre Unitary Ensemble (LUE), the celebrated Tracy-Widom II distribution [29], denoted as $F_{2}$, is given by a integral involving the Hastings-McLeod solution of the Painlevé II equation. For the GUE, it can be immediately seen via a change of variables that the analogous result holds for the smallest eigenvalue. These distributions emerge after centering the extreme eigenvalues at $\pm \sqrt{2 n}$, the edges of the GUE spectrum, followed by a scaling with respect to the density at the soft edge; see [29] for the original derivation of this particular $P_{I I}$. In [30] a finite $n$ version of the result was found, which turns out to be a $P_{I V}$, with the limiting $F_{2}$ after the above centering and scaling of the largest eigenvalues. For LUE, the distribution is obtained after centering the largest eigenvalues at $4 n$ and a scaling at the soft edge of the LUE spectrum. The finite $n$ analogue in this case was found to be a $P_{V}$ [30].

In this paper we consider a related problem. We are concerned with the probability that all the eigenvalues are in an interval $(a, b)$. This is of course equivalent to the probability that the largest eigenvalue is less than $b$ and the smallest one greater than $a$. It turns out that this probability is related to another expression that is the solution of a nonlinear partial differential equation (pde). In the GUE case, this pde maybe interpreted as a two variable version of a Painlevé IV $\sigma$-form, since in the limit $a$ fixed, $b \rightarrow \infty$ or $b$ fixed, $a \rightarrow-\infty$, the pde reduces to the ode corresponding to the "left" or "right" edge version of $P_{I V}$. We have a similar interpretation for the pde derived in the LUE case as a two variable version of a Painlevé V $\sigma$-form. See also [2] where the authors derived pdes for the logarithm of this probability which are related to KP equations using vertex operators and the associated Virasoro constraint [1].

Our method is based on a ladder operators formalism for orthogonal polynomials [13] and the associated compatibility conditions. This is, by now, a well-known method that has been applied to obtain exact solutions in a classical case [14] and adapted to orthogonal polynomials on the unit circle [4] intimately related to the theory of Toeplitz matrices. One may find in [16] a list of references on this formalism. Recent examples on the applications of the ladder operators with the resulting Painlevé equations can be found in [5, 11, 12, 17, 19, 20, 22]; see also [18] for an application to the information theory of multiple input and multiple output wireless communications which involves certain deformation of the LUEs. In particular, a comparison between the ladder operator theory and the isomonodromy theory of Jimbo-Miwa-Ujimo [26] is carried out in [16] and [22] for different specific Hermitian random ensembles. The extension of ladder operators to discrete orthogonal polynomials and $q$-orthogonal polynomials is given in [24] and [15], respectively. From these extensions, 
it is shown that the recurrence coefficients of certain discrete or $q$-orthogonal polynomials are related to the discrete or $q$-Painlevé equations, respectively. We refer to [7, 8, 9, 23] for investigations of this aspect.

Recently, based entirely on the estimate on the integral operator in a Fredholm expansion, the authors of [6] showed that the extreme eigenvalues of GUE, when suitably centered and scaled, are asymptotically independent random variables. As an application of our result, we give a proof that is an analytic counter-part of this probabilistic version. Our strategy is to scale the two variables in the pde obtained near both edges of the GUE spectrum, which will lead to a limiting pde. It turns out that the solution to the limiting pde has a particular form which implies that the joint probability density is a product of two independent densities, that is, there is asymptotic independence. With different and delicate scalings, we show that the solution of limiting pde in LUE case is also asymptotically equal to the sum of Tracy-Widom left and right distributions.

The rest of this paper is organized as follows. In section 2, we give a summary of the ladder operators method, the associated compatibility conditions and a summation identity. From these conditions, we derive a system of non-linear difference equations satisfied by auxiliary variables that appear naturally in this approach. These difference equations will be instrumental in our further derivation of the pdes. We study GUE in section 3 and LUE in section 4. As an application of our result, we give an analytic proof that the extreme eigenvalues of GUE and LUE, when suitably centered and scaled, are asymptotic independent.

\section{Preliminaries}

In the theory of Hermitian random matrices, one encounters the following (un-normalized) joint probability density of eigenvalues $\left\{x_{j}\right\}_{j=1}^{n}$ :

$$
p\left(x_{1}, \ldots, x_{n}\right)=\prod_{1 \leq i<j \leq n}\left[\Delta_{n}(x)\right]^{2} \prod_{k=1}^{n} w\left(x_{k}\right)
$$

where

$$
\Delta_{n}(x):=\prod_{1 \leq j<i \leq n}\left(x_{i}-x_{j}\right)
$$

and $w$ is a weight function defined on an interval $I \subseteq \mathbb{R}$. The GUE corresponds to $w(x)=\mathrm{e}^{-x^{2}}$ and $I=\mathbb{R}$, while the LUE corresponds to $w(x)=x^{\alpha} \mathrm{e}^{-x}, \alpha>0$ and $I=(0, \infty)$. In what follows everything depends on $n$, but the dependence will only be displayed when it is easy to do so or needed for clarification. 
Denote by $\operatorname{Prob}(n, a, b)$ the probability that all eigenvalues lie in an interval $(a, b) \subset I$. We then have

$$
\operatorname{Prob}(n, a, b)=\frac{\int_{(a, b)^{n}}\left[\Delta_{n}(x)\right]^{2} \prod_{k=1}^{n} w\left(x_{k}\right) d x_{k}}{\int_{I^{n}}\left[\Delta_{n}(x)\right]^{2} \prod_{k=1}^{n} w\left(x_{k}\right) d x_{k}} .
$$

It is a well-known fact (cf. [28]) that the multiple integrals in (2.3) can be expressed as determinant of Hankel matrix generated by the weight function $w$. The moments of the weight are defined by

$$
\mu_{j}(a, b):=\int_{a}^{b} x^{j} w(x) d x, \quad j=0,1, \ldots,
$$

and the Hankel determinant is the determinant of the moment matrix $\left(\mu_{j+k}\right)_{j, k=0}^{n-1}$ :

$$
\begin{aligned}
D_{n}(a, b) & :=\operatorname{det}\left(\mu_{j+k}(a, b)\right)_{j, k=0}^{n-1} \\
& =\prod_{j=0}^{n-1} h_{j}(a, b) \\
& =\frac{1}{n !} \int_{(a, b)^{n}}\left[\Delta_{n}(x)\right]^{2} \prod_{k=1}^{n} w\left(x_{k}\right) d x_{k} .
\end{aligned}
$$

Here, $h_{i}(a, b)$ is the square of the $L^{2}$ norm of the (monic) polynomials orthogonal with respect to $w$ over $(a, b)$, i.e.,

$$
\int_{a}^{b} P_{i}(x, a, b) P_{j}(x, a, b) w(x) d x=h_{i}(a, b) \delta_{i, j} .
$$

The monic polynomials $P_{n}(z, a, b)$ are normalized as

$$
P_{n}(z, a, b)=z^{n}+\mathrm{p}_{1}(n, a, b) z^{n-1}+\ldots+P_{n}(0, a, b) .
$$

Therefore,

$$
\operatorname{Prob}(n, a, b)=\frac{D_{n}(a, b)}{D_{n}(I)}=\prod_{j=0}^{n-1} \frac{h_{j}(a, b)}{h_{j}(I)} .
$$

It is then clear that in order to compute the probability $\operatorname{Prob}(n, a, b)$, we need to compute the product of the norms $h_{i}(a, b)$ and thus we need information about the orthogonal polynomials. According to the general theory of orthogonal polynomials, an immediate consequence of (2.6) is the three terms recurrence relations:

$$
z P_{n}(z, a, b)=P_{n+1}(z, a, b)+\alpha_{n}(a, b) P_{n}(z, a, b)+\beta_{n}(a, b) P_{n-1}(z, a, b)
$$

with the initial conditions

$$
P_{0}(z)=1, \quad \beta_{0} P_{-1}(z)=0 .
$$


An easy consequence of the recurrence relation is

$$
\alpha_{n}(a, b)=\mathrm{p}_{1}(n, a, b)-\mathrm{p}_{1}(n+1, a, b)
$$

In the next two sections, we will give an account for a recursive algorithm for the determination of the recurrence coefficients $\alpha_{n}, \beta_{n}$ in the GUE and LUE, respectively, based on a pair of ladder operators and the associated supplementary conditions. It will become clear that the determination of $\alpha_{n}$ and $\beta_{n}$ will yield the necessary information to find conditions on $h_{n}(a, b)$. The following three lemmas can be found in, for example, [16] and the extensive references therein. For convenience, we suppress the $a, b$ dependence in $\alpha_{n}, \beta_{n}$ and $h_{n}$ in these lemmas.

Lemma 1. Suppose that $w$ is such that the moments

$$
\mu_{i}(a, b):=\int_{a}^{b} x^{i} w(x) d x, \quad i=0,1, \ldots
$$

exist and that $v=-\ln w$ has a derivative in some Lipshitz class with positive exponent. The lowering and raising operators satisfy the following:

$$
\begin{aligned}
P_{n}^{\prime}(z, a, b) & =-B_{n}(z, a, b) P_{n}(z, a, b)+\beta_{n} A_{n}(z, a, b) P_{n-1}(z, a, b), \\
P_{n-1}^{\prime}(z, a, b) & =\left[B_{n}(z, t)+\vee(z)\right] P_{n-1}(z, a, b)-A_{n-1}(z, a, b) P_{n}(z, a, b),
\end{aligned}
$$

where

$$
\begin{gathered}
A_{n}(z, a, b):=\left.\frac{w(y) P_{n}^{2}(y, a, b)}{h_{n}(y-z)}\right|_{a} ^{b}+\frac{1}{h_{n}} \int_{a}^{b} \frac{V(z)-\vee(y)}{z-y} P_{n}^{2}(y, a, b) w(y) d y \\
B_{n}(z, a, b):=\left.\frac{w(y) P_{n}(y, a, b) P_{n-1}(y, a, b)}{h_{n-1}(y-z)}\right|_{a} ^{b}+\frac{1}{h_{n-1}} \int_{a}^{b} \frac{V(z)-\vee(y)}{z-y} P_{n}(y, a, b) P_{n-1}(y, a, b) w(y) d y .
\end{gathered}
$$

A direct calculation produces two fundamental supplementary (compatibility) conditions valid for all $z$ and these are stated in two different forms in the next lemma.

Lemma 2. The functions $A_{n}(z, a, b)$ and $B_{n}(z, a, b)$ satisfy the conditions:

$$
\begin{gathered}
B_{n+1}(z, a, b)+B_{n}(z, a, b)=\left(z-\alpha_{n}\right) A_{n}(z, a, b)-\vee(z) \\
1+\left(z-\alpha_{n}\right)\left(B_{n+1}(z, a, b)-B_{n}(z, a, b)\right)=\beta_{n+1} A_{n+1}(z, a, b)-\beta_{n} A_{n-1}(z, a, b) .
\end{gathered}
$$

It turns out that there is an equation which gives better insight into the $\alpha_{n}$ and $\beta_{n}$ if $\left(S_{1}\right)$ and $\left(S_{2}\right)$ are suitably combined. 
Lemma 3. The functions $A_{n}(z, a, b), B_{n}(z, a, b)$ and

$$
\sum_{j=0}^{n-1} A_{j}(z, a, b)
$$

satisfy the condition:

$$
B_{n}^{2}(z, a, b)+\mathcal{V}(z) B_{n}(z, a, b)+\sum_{j=0}^{n-1} A_{j}(z, a, b)=\beta_{n} A_{n}(z, a, b) A_{n-1}(z, a, b)
$$

\section{Studies of Gaussian Unitary Ensembles}

It is the aim of this section to show that a quantity associated with $\operatorname{Prob}(n, a, b)$ defined in (2.3) Satisfies a nonlinear pde for GUE via the ladder operators introduced in the previous section. To this end, we recall that in the present case $w(x)=\mathrm{e}^{-x^{2}}$ and $I=\mathbb{R}$. Hence, an appeal to lemma 1 gives

$$
\begin{aligned}
& A_{n}(z, a, b)=\frac{R_{n, b}}{z-b}+\frac{R_{n, a}}{z-a}+2, \\
& B_{n}(z, a, b)=\frac{r_{n, b}}{z-b}+\frac{r_{n, a}}{z-a}
\end{aligned}
$$

where

$$
\begin{aligned}
R_{n, b} & =-\mathrm{e}^{-b^{2}} \frac{P_{n}^{2}(b, a, b)}{h_{n}(a, b)}, \\
R_{n, a} & =\mathrm{e}^{-a^{2}} \frac{P_{n}^{2}(a, a, b)}{h_{n}(a, b)}, \\
r_{n, b} & =-\mathrm{e}^{-b^{2}} \frac{P_{n}(b, a, b) P_{n-1}(b, a, b)}{h_{n-1}(a, b)}, \\
r_{n, a} & =\mathrm{e}^{-a^{2}} \frac{P_{n}(a, a, b) P_{n-1}(a, a, b)}{h_{n-1}(a, b)} .
\end{aligned}
$$

Substituting these into $\left(S_{1}\right)$ implies

$$
\begin{aligned}
R_{n, a}+R_{n, b} & =2 \alpha_{n}, \\
r_{n+1, b}+r_{n, b} & =\left(b-\alpha_{n}\right) R_{n, b}, \\
r_{n+1, a}+r_{n, a} & =\left(a-\alpha_{n}\right) R_{n, a} ;
\end{aligned}
$$


while into $\left(S_{2}^{\prime}\right)$,

$$
\begin{aligned}
\beta_{n} & =\frac{n}{2}+\frac{r_{n, a}}{2}+\frac{r_{n, b}}{2}, \\
r_{n, a}^{2} & =\beta_{n} R_{n, a} R_{n-1, a}, \\
r_{n, b}^{2} & =\beta_{n} R_{n, b} R_{n-1, b}, \\
2 \frac{r_{n, b} r_{n, a}}{b-a}+2 b r_{n, b}+\sum_{j=0}^{n-1} R_{j, b} & =\beta_{n}\left[\frac{R_{n, b} R_{n-1, a}+R_{n-1, b} R_{n, a}}{b-a}+2\left(R_{n-1, b}+R_{n, b}\right)\right], \\
2 \frac{r_{n, b} r_{n, a}}{a-b}+2 a r_{n, a}+\sum_{j=0}^{n-1} R_{j, a} & =\beta_{n}\left[\frac{R_{n, a} R_{n-1, b}+R_{n-1, a} R_{n, b}}{a-b}+2\left(R_{n-1, a}+R_{n, a}\right)\right] .
\end{aligned}
$$

The sum of the last two equations gives

$$
\begin{aligned}
2 b r_{n, b}+2 a r_{n, a}+\sum_{j=0}^{n-1}\left(R_{j, a}+R_{j, b}\right) & =2 \beta_{n}\left(R_{n-1, b}+R_{n, b}+R_{n-1, a}+R_{n, a}\right) \\
& =2 \beta_{n}\left(R_{n, a}+R_{n, b}\right)+2 \frac{r_{n, a}^{2}}{R_{n, a}}+2 \frac{r_{n, b}^{2}}{R_{n, b}}
\end{aligned}
$$

where we have made use of (3.11) and (3.12) to eliminate the terms $R_{n-1, a}, R_{n-1, b}$ and arrive at the last equation.

\subsection{Toda evolution}

Before coming to the derivation of pde, we first establish some connections between $\beta_{n}$, $\mathrm{p}_{1}(n, a, b)$ and the auxiliary constants $r_{n, a}, r_{n, b}, R_{n, a}$ and $R_{n, b}$, which will lead to a two variable analogue of the Toda equations for the recurrence coefficients $\alpha_{n}$ and $\beta_{n}$. These relations will be helpful in our further analysis.

We start with taking the partial derivative of $h_{n}$ with respect to $b$ in (2.6), which gives

$$
\partial_{b} h_{n}=\mathrm{e}^{-b^{2}} P_{n}^{2}(b, a, b),
$$

and consequently

$$
\partial_{b} \ln h_{n}=-R_{n, b} .
$$

Since $\beta_{n}=h_{n} / h_{n-1}$, we find,

$$
\frac{1}{\beta_{n}} \partial_{b} \beta_{n}=R_{n-1, b}-R_{n, b},
$$


and a similar computation yields

$$
\frac{1}{\beta_{n}} \partial_{a} \beta_{n}=R_{n-1, a}-R_{n, a} .
$$

Again taking partial derivatives with respect to $b$ on both sides of the equation

$$
0=\int_{a}^{b} P_{n}(x, a, b) P_{n-1}(x, a, b) \mathrm{e}^{-x^{2}} d x
$$

produces

$$
\begin{aligned}
0 & =P_{n}(b, a, b) P_{n-1}(b, a, b) \mathrm{e}^{-b^{2}}+\int_{a}^{b}\left[\partial_{b} \mathrm{p}_{1}(n, a, b) x^{n-1}+\cdots\right] P_{n-1}(x, a, b) \mathrm{e}^{-x^{2}} d x \\
& =P_{n}(b, a, b) P_{n-1}(b, a, b) \mathrm{e}^{-b^{2}}+\partial_{b} \mathrm{p}_{1}(n, a, b) h_{n-1}(a, b),
\end{aligned}
$$

and consequently

$$
\partial_{b} \mathrm{p}_{1}(n, a, b)=-\mathrm{e}^{-b^{2}} \frac{P_{n}(b, a, b) P_{n-1}(b, a, b)}{h_{n-1}(a, b)}=r_{n, b} .
$$

A similar computation yields

$$
\partial_{a} \mathrm{p}_{1}(n, a, b)=\mathrm{e}^{-a^{2}} \frac{P_{n}(a, a, b) P_{n-1}(a, a, b)}{h_{n-1}(a, b)}=r_{n, a} .
$$

Now, we are ready to prove:

Lemma 4. For GUE, we have

$$
\begin{aligned}
\frac{1}{\beta_{n}}\left(\partial_{a}+\partial_{b}\right) \beta_{n} & =2\left(\alpha_{n-1}-\alpha_{n}\right), \\
\left(\partial_{a}+\partial_{b}\right) \alpha_{n} & =2\left(\beta_{n}-\beta_{n+1}\right)-1 .
\end{aligned}
$$

Proof. Taking a sum of (3.18) and (3.19), the equation (3.22) is immediate from (3.7). To estblish (3.23), we note from the sum of (3.20) and (3.21) that

$$
\left(\partial_{a}+\partial_{b}\right) \mathrm{p}_{1}(n, a, b)=r_{n, a}+r_{n, b}=2 \beta_{n}-n,
$$

where the last equality follows from (3.10). This, together with (2.11), gives us (3.23).

The equations (3.22) and (3.23) constitute a two variable version of the Toda equations. 


\subsection{Derivation of partial differential equation}

To this end, we set

$$
H_{n}(a, b):=\left(\partial_{a}+\partial_{b}\right) \ln D_{n}(a, b),
$$

where $D_{n}$ is the Hankel determinant defined in (2.5) associated with GUE. It is the aim of this section to derive a pde satisfied by $H_{n}$. Our strategy is to construct a system of partial differential equations in the functions $r_{n, a}, r_{n, b}, R_{n, b}$ and $R_{n, a}$, because they provide a direct link to $H_{n}$.

To see this, note that

$$
\ln D_{n}(a, b)=\sum_{j=0}^{n-1} \ln h_{j}(a, b),
$$

and thus

$$
\begin{aligned}
\partial_{a} \ln D_{n}(a, b) & =\sum_{j=0}^{n-1} \partial_{a} \ln h_{j}(a, b)=-\sum_{j=0}^{n-1} R_{j, a} \\
\partial_{b} \ln D_{n}(a, b) & =\sum_{j=0}^{n-1} \partial_{b} \ln h_{j}(a, b)=-\sum_{j=0}^{n-1} R_{j, b} .
\end{aligned}
$$

Recall that we have from (3.7)

$$
R_{n, b}+R_{n, a}=2 \alpha_{n},
$$

and that from (2.11)

$$
\alpha_{n}(a, b)=\mathrm{p}_{1}(n, a, b)-\mathrm{p}_{1}(n+1, a, b) .
$$

Therefore we have that

$$
\sum_{j=0}^{n-1}\left(R_{j, b}+R_{j, a}\right)=2 \sum_{j=0}^{n-1} \alpha_{j}(a, b)=-2 \mathrm{p}_{1}(n, a, b) .
$$

A combination of (3.24), (3.26) and (3.27) gives us

$$
\begin{aligned}
H_{n} & =\left(\partial_{a}+\partial_{b}\right) \ln D_{n}=-\sum_{j=0}^{n-1}\left(R_{j, a}+R_{j, b}\right)=2 \mathrm{p}_{1}(n, a, b) \\
& =2 a r_{n, a}+2 b r_{n, b}-2 \frac{r_{n, a}^{2}}{R_{n, a}}-2 \frac{r_{n, b}^{2}}{R_{n, b}}-2 \beta_{n}\left(R_{n, a}+R_{n, b}\right),
\end{aligned}
$$


where the second equality of (3.29) follows from (3.15). In view of (3.20) and (3.21), we note that

$$
\partial_{a} H_{n}=2 r_{n, a}, \quad \partial_{b} H_{n}=2 r_{n, b} .
$$

From (3.19), (3.18), (3.11) and (3.12), we have

$$
\begin{aligned}
& \partial_{a} \beta_{n}=\frac{r_{n, a}^{2}}{R_{n, a}}-\beta_{n} R_{n, a} \\
& \partial_{b} \beta_{n}=\frac{r_{n, b}^{2}}{R_{n, b}}-\beta_{n} R_{n, b} .
\end{aligned}
$$

This, together with (3.10), implies

$$
\begin{aligned}
& \frac{1}{2} \partial_{a}\left(r_{n, a}+r_{n, b}\right)=\frac{r_{n, a}^{2}}{R_{n, a}}-\left(\frac{n}{2}+\frac{r_{n, a}+r_{n, b}}{2}\right) R_{n, a} \\
& \frac{1}{2} \partial_{b}\left(r_{n, a}+r_{n, b}\right)=\frac{r_{n, b}^{2}}{R_{n, b}}-\left(\frac{n}{2}+\frac{r_{n, a}+r_{n, b}}{2}\right) R_{n, b} .
\end{aligned}
$$

Expressing $r_{n, a}$ and $r_{n, a}$ in terms of the partial derivatives of $H_{n}$, we have

$$
\begin{aligned}
& \partial_{a}^{2} H_{n}+\partial_{a} \partial_{b} H_{n}=\frac{\left(\partial_{a} H_{n}\right)^{2}}{R_{n, a}}-\left(2 n+\partial_{a} H_{n}+\partial_{b} H_{n}\right) R_{n, a}, \\
& \partial_{b}^{2} H_{n}+\partial_{a} \partial_{b} H_{n}=\frac{\left(\partial_{b} H_{n}\right)^{2}}{R_{n, b}}-\left(2 n+\partial_{a} H_{n}+\partial_{b} H_{n}\right) R_{n, b} .
\end{aligned}
$$

We may consider (3.33) and (3.34) as quadratic equations in $R_{n, a}$ and $R_{n, b}$. Solving for them and substituting into (3.29), we find, after some simplification,

$$
\begin{aligned}
2 b \partial_{b} H_{n}+2 a \partial_{a} H_{n}-2 H_{n}= & \sqrt{\left(\partial_{a}^{2} H_{n}+\partial_{a} \partial_{b} H_{n}\right)^{2}+4\left(\partial_{a} H_{n}\right)^{2}\left(2 n+\partial_{a} H_{n}+\partial_{b} H_{n}\right)} \\
& -\sqrt{\left(\partial_{b}^{2} H_{n}+\partial_{a} \partial_{b} H_{n}\right)^{2}+4\left(\partial_{b} H_{n}\right)^{2}\left(2 n+\partial_{a} H_{n}+\partial_{b} H_{n}\right)} .
\end{aligned}
$$

(Note that in the above the signs of the square roots are determined by the signs of $R_{n, a}$ and $R_{n, b}$. The former is positive and the latter is negative and the term $2 n+\partial_{a} H_{n}+\partial_{b} H_{n}$ is also positive since it is the same as $4 \beta_{n}$.) After clearing the square roots, we obtain that the function $H_{n}$ defined in (3.24) satisfies the following pde:

$$
\begin{gathered}
\left(\left(2 b \partial_{b} H_{n}+2 a \partial_{a} H_{n}-2 H_{n}\right)^{2}-\left(\left(\partial_{a}^{2} H_{n}+\partial_{a} \partial_{b} H_{n}\right)^{2}+4\left(\partial_{a} H_{n}\right)^{2}\left(2 n+\partial_{a} H_{n}+\partial_{b} H_{n}\right)\right)\right. \\
\left.-\left(\left(\partial_{b}^{2} H_{n}+\partial_{a} \partial_{b} H_{n}\right)^{2}+4\left(\partial_{b} H_{n}\right)^{2}\left(2 n+\partial_{a} H_{n}+\partial_{b} H_{n}\right)\right)\right)^{2} \\
=4\left(\left(\partial_{a}^{2} H_{n}+\partial_{a} \partial_{b} H_{n}\right)^{2}+4\left(\partial_{a} H_{n}\right)^{2}\left(2 n+\partial_{a} H_{n}+\partial_{b} H_{n}\right)\right) \\
\times\left(\left(\partial_{b}^{2} H_{n}+\partial_{a} \partial_{b} H_{n}\right)^{2}+4\left(\partial_{b} H_{n}\right)^{2}\left(2 n+\partial_{a} H_{n}+\partial_{b} H_{n}\right)\right)
\end{gathered}
$$


In our approach, the end points $a$ and $b$ are the "times", although they play a distinct role from those in a two variable generalization of Painlevé IV [31].

Suppose $H_{n}$ is independent of $a$, the equation (3.36) reduce to

$$
\left(\partial_{b}^{2} H_{n}\right)^{2}=4\left(b \partial_{b} H_{n}-H_{n}\right)^{2}-4\left(\partial_{b} H_{n}\right)^{2}\left(2 n+\partial_{b} H_{n}\right)
$$

which actually is the Okamoto-Jimbo-Miwa $\sigma$-form of the Painlevé IV equation [25]:

$$
\left(\sigma^{\prime \prime}\right)^{2}=4\left(z \sigma^{\prime}-\sigma\right)^{2}-4\left(\sigma^{\prime}+\nu_{0}\right)\left(\sigma^{\prime}+\nu_{1}\right)\left(\sigma^{\prime}+\nu_{2}\right)
$$

with

$$
\nu_{0}=2 n, \quad \nu_{1}=\nu_{2}=0 .
$$

The same conclusion holds if $H_{n}$ is independent of $b$.

Finally, it may be obvious, but worth pointing out that the solution $H_{n}$ to the pde along with initial conditions does indeed yield the desired probability. This is because

$$
\ln \operatorname{Prob}(n, a, b)=\int_{0}^{a} H_{n}(t, t+b-a) d t+\ln \operatorname{Prob}(n, 0, b-a)
$$

\subsection{Asymptotic independence of the extreme eigenvalues in GUE}

As an application of the pde derived in section 3.2, we show in this section that the extreme eigenvalues of GUE, when suitably centered and scaled, are asymptotic independent, i.e.,

$$
\begin{aligned}
& \lim _{n \rightarrow \infty} \operatorname{Prob}\left(n,\left(\lambda_{\min }+\sqrt{2 n}\right) n^{1 / 6} / c>x,\left(\sqrt{2 n}-\lambda_{\max }\right) n^{1 / 6} / c<y\right) \\
& =\lim _{n \rightarrow \infty} \operatorname{Prob}\left(\left(\lambda_{\min }+\sqrt{2 n}\right) n^{1 / 6} / c>x\right) \lim _{n \rightarrow \infty} \operatorname{Prob}\left(\left(\sqrt{2 n}-\lambda_{\max }\right) n^{1 / 6} / c<y\right),
\end{aligned}
$$

where $\lambda_{\min }\left(\lambda_{\max }\right)$ denotes the smallest (largest) eigenvalue.

Our method is to scale $a$ and $b$ near the edges of the spectrum and compute asymptotically the resulting pde. For this purpose, we let

$$
a=-\sqrt{2 n}+c \frac{x}{n^{1 / 6}}, \quad b=\sqrt{2 n}-c \frac{y}{n^{1 / 6}},
$$

with $c>0$, and note that

$$
\partial_{a}=\frac{n^{1 / 6}}{c} \partial_{x}, \quad \partial_{b}=-\frac{n^{1 / 6}}{c} \partial_{y}
$$

Recall

$$
H_{n}(a, b)=\left(\partial_{a}+\partial_{b}\right) \ln D_{n}(a, b)=\left(\partial_{a}+\partial_{b}\right) \ln \operatorname{Prob}(n, a, b),
$$


and in the $x, y$ variables this becomes

$$
\frac{c}{n^{1 / 6}} H_{n}\left(-\sqrt{2 n}+c \frac{x}{n^{1 / 6}}, \sqrt{2 n}-c \frac{y}{n^{1 / 6}}\right)=\left(\partial_{x}-\partial_{y}\right) \ln D_{n} .
$$

Let

$$
\widetilde{H}(x, y, n):=\frac{c}{n^{1 / 6}} H_{n}\left(-\sqrt{2 n}+c \frac{x}{n^{1 / 6}}, \sqrt{2 n}-c \frac{y}{n^{1 / 6}}\right) .
$$

After substituting the change of variables (3.2), the leading term of (3.35) is of order $n^{4 / 3}$ and produces the limiting pde

$$
\begin{aligned}
& -8 \sqrt{2} c^{3} \widetilde{H} \partial_{y} \widetilde{H} \partial_{x} \widetilde{H}+8 \sqrt{2} c^{3} y\left(\partial_{y} \widetilde{H}\right)^{2} \partial_{x} \widetilde{H}-4\left(\partial_{y} \widetilde{H}\right)^{3} \partial_{x} \widetilde{H}+\partial_{x} \widetilde{H}\left(\partial_{y}^{2} \widetilde{H}-\partial_{x} \partial_{y} \widetilde{H}\right)^{2} \\
& +\partial_{y} \widetilde{H}\left(8 \sqrt{2} c^{3} x\left(\partial_{x} \widetilde{H}\right)^{2}+4\left(\partial_{x} \widetilde{H}\right)^{3}+\left(\partial_{x} \partial_{y} \widetilde{H}-\partial_{x}^{2} \widetilde{H}\right)^{2}\right)=0 .
\end{aligned}
$$

To ascertain whether the scaled smallest and largest eigenvalues may be described by their respective Tracy-Widom law for the extreme eigenvalues, we make use of a factorization ansatz,

$$
\widetilde{H}(x, y)=f(x)+g(y)
$$

where $f(x)$ and $g(y)$ satisfy the $\sigma$-form of a particular Painlevé II. That is,

$$
\begin{aligned}
& \frac{1}{4}\left(f^{\prime \prime}(x)\right)^{2}=2 \sqrt{2} c^{3} f(x) f^{\prime}(x)-2 \sqrt{2} c^{3} x\left(f^{\prime}(x)\right)^{2}-\left(f^{\prime}(x)\right)^{3}, \\
& \frac{1}{4}\left(g^{\prime \prime}(y)\right)^{2}=2 \sqrt{2} c^{3} g(y) g^{\prime}(y)-2 \sqrt{2} c^{3} y\left(g^{\prime}(y)\right)^{2}+\left(g^{\prime}(y)\right)^{3} .
\end{aligned}
$$

We use this ansatz because if $x=-\infty$ or $y=\infty$, then the functions $f$ and $g$ yield the correct solution of the pde and we believe that the solution should be their sum. Indeed this is true. An simple computation shows that (3.5) is satisfied identically.

Now, we set

$$
\mathrm{P}(x, y)=\lim _{n \rightarrow \infty} \operatorname{Prob}\left(n,\left(\lambda_{\min }+\sqrt{2 n}\right) n^{1 / 6} / c>x,\left(\sqrt{2 n}-\lambda_{\max }\right) n^{1 / 6} / c<y\right) .
$$

Recall that

$$
\left(\partial_{x}-\partial_{y}\right) \ln \mathrm{P}(x, y)=\lim _{n \rightarrow \infty} \widetilde{H}(x, y, n) .
$$

Therefore the general solution of $\mathrm{P}(x, y)$ is of this form

$$
\mathrm{P}(x, y)=F(x) G(y) \exp [\Psi(x+y)]
$$

where

$$
\begin{aligned}
& F(x)=\lim _{n \rightarrow \infty} \operatorname{Prob}\left(\left(\lambda_{\min }+\sqrt{2 n}\right) n^{1 / 6} / c>x\right), \\
& G(y)=\lim _{n \rightarrow \infty} \operatorname{Prob}\left(\left(\sqrt{2 n}-\lambda_{\max }\right) n^{1 / 6} / c<y\right),
\end{aligned}
$$


and $\Psi$ is an arbitrary $C^{1}$ function. In view of (3.1), it remains to show $\Psi \equiv 0$. To see this, note that

$$
\begin{array}{ll}
\lim _{x \rightarrow-\infty} F(x)=1, & \lim _{x \rightarrow \infty} F(x)=0, \\
\lim _{y \rightarrow-\infty} G(y)=0, & \lim _{y \rightarrow \infty} G(y)=1,
\end{array}
$$

and

$$
\lim _{x \rightarrow-\infty, y \rightarrow \infty} \mathrm{P}(x, y)=1 .
$$

Take a fixed $z$ and let $x+y=z$. We see that

$$
\lim _{x \rightarrow-\infty} \mathrm{P}(x, z-x)=1=\exp [\Psi(z)]
$$

for all $z$. Hence $\Psi \equiv 0$.

An operator-theoretic proof of the asymptotic independence which also provide the rate of convergence to the factored Tracy-Widom distributions can be found in [10].

\section{Studies of Laguerre Unitary Ensembles}

This section is devoted to the study of LUE. Hence, it is understood that all the notations $h_{n}(a, b), \alpha_{n}, \beta_{n}, \mathrm{p}_{1}(n, a, b)$, etc. in this section are now associated with $P_{n}(z, a, b)$ defined in (2.6) with $w(x)=x^{\alpha} \mathrm{e}^{-x}$ and $I=(0, \infty)$. We will apply a similar theme to the LUE case as in the GUE case.

By applying lemma 1 to the Laguerre weight $w(x)=x^{\alpha} \mathrm{e}^{-x}$, it is readily seen that

$$
\begin{aligned}
& A_{n}(z, a, b)=\frac{R_{n}}{z}+\frac{R_{n, a}}{z-a}+\frac{R_{n, b}}{z-b}, \\
& B_{n}(z, a, b)=\frac{r_{n}}{z}+\frac{r_{n, a}}{z-a}+\frac{r_{n, b}}{z-b},
\end{aligned}
$$


where

$$
\begin{aligned}
R_{n} & =\frac{\alpha}{h_{n}(a, b)} \int_{a}^{b} P_{n}^{2}(y, a, b) y^{\alpha-1} \mathrm{e}^{-y} d y \\
R_{n, a} & =a^{\alpha} \mathrm{e}^{-a} \frac{P_{n}^{2}(a, a, b)}{h_{n}(a, b)} \\
R_{n, b} & =-b^{\alpha} \mathrm{e}^{-b} \frac{P_{n}^{2}(b, a, b)}{h_{n}(a, b)} \\
r_{n} & =\frac{\alpha}{h_{n-1}(a, b)} \int_{a}^{b} P_{n}(y, a, b) P_{n-1}(y, a, b) y^{\alpha-1} \mathrm{e}^{-y} d y \\
r_{n, a} & =a^{\alpha} \mathrm{e}^{-a} \frac{P_{n}(a, a, b) P_{n-1}(a, a, b)}{h_{n-1}(a, b)} \\
r_{n, b} & =-b^{\alpha} \mathrm{e}^{-b} \frac{P_{n}(b, a, b) P_{n-1}(b, a, b)}{h_{n-1}(a, b)} .
\end{aligned}
$$

Substituting the above formulas into $\left(S_{1}\right)$, we obtain

$$
\begin{aligned}
R_{n}+R_{n, a}+R_{n, b} & =1, \\
r_{n}+r_{n+1} & =\alpha-\alpha_{n} R_{n}, \\
r_{n, a}+r_{n+1, a} & =\left(a-\alpha_{n}\right) R_{n, a}, \\
r_{n, b}+r_{n+1, b} & =\left(b-\alpha_{n}\right) R_{n, b} .
\end{aligned}
$$

From $\left(S_{2}^{\prime}\right)$, we find,

$$
\begin{gathered}
r_{n}^{2}-\alpha r_{n}=\beta_{n} R_{n} R_{n-1}, \\
r_{n, a}^{2}=\beta_{n} R_{n-1, a} R_{n, a}, \\
r_{n, b}^{2}=\beta_{n} R_{n-1, b} R_{n, b}, \\
-2 \frac{r_{n} r_{n, a}}{a}-2 \frac{r_{n} r_{n, b}}{b}+r_{n}+\alpha \frac{r_{n, a}}{a}+\alpha \frac{r_{n, b}}{b}+\sum_{j=0}^{n-1} R_{j} \\
=-\beta_{n}\left(\frac{R_{n-1, a} R_{n}+R_{n-1} R_{n, a}}{a}+\frac{R_{n-1, b} R_{n}+R_{n-1} R_{n, b}}{b}\right), \\
2 \frac{r_{n} r_{n, a}}{a}+2 \frac{r_{n, a} r_{n, b}}{a-b}+r_{n, a}-\alpha \frac{r_{n, a}}{a}+\sum_{j=0}^{n-1} R_{j, a} \\
=\beta_{n}\left(\frac{R_{n-1, a} R_{n}+R_{n-1} R_{n, a}}{a}+\frac{R_{n-1, a} R_{n, b}+R_{n-1, b} R_{n, a}}{a-b}\right),
\end{gathered}
$$




$$
\begin{aligned}
& 2 \frac{r_{n} r_{n, b}}{b}+2 \frac{r_{n, a} r_{n, b}}{b-a}+r_{n, b}-\alpha \frac{r_{n, b}}{b}+\sum_{j=0}^{n-1} R_{j, b} \\
& =\beta_{n}\left(\frac{R_{n-1, b} R_{n}+R_{n-1} R_{n, b}}{b}+\frac{R_{n-1, a} R_{n, b}+R_{n-1, b} R_{n, a}}{b-a}\right) .
\end{aligned}
$$

From $\left(S_{2}\right)$, we find

$$
\begin{aligned}
1+r_{n+1}-r_{n}+r_{n+1, a}-r_{n, a}+r_{n+1, b}-r_{n, b} & =0, \\
\alpha_{n}\left(r_{n}-r_{n+1}\right) & =\beta_{n+1} R_{n+1}-\beta_{n} R_{n-1}, \\
\left(a-\alpha_{n}\right)\left(r_{n+1, a}-r_{n, a}\right) & =\beta_{n+1} R_{n+1, a}-\beta_{n} R_{n-1, a}, \\
\left(b-\alpha_{n}\right)\left(r_{n+1, b}-r_{n, b}\right) & =\beta_{n+1} R_{n+1, b}-\beta_{n} R_{n-1, b} .
\end{aligned}
$$

The sum of (4.16)-(4.18) yields

$$
r_{n}+r_{n, a}+r_{n, b}+n=0,
$$

where we have made use of (4.9). This equation can also be obtained by a telescopic sum of (4.19). Summing (4.10)-(4.12), we see from (4.9) and (4.23) that

$$
\alpha_{n}=\alpha+a R_{n, a}+b R_{n, b}+2 n+1 .
$$

By (4.17)-(4.18), it is easily seen that

$$
\begin{aligned}
& a \sum_{j=0}^{n-1} R_{j, a}+b \sum_{j=1}^{n-1} R_{j, b} \\
& =\beta_{n}\left(\left(R_{n-1, a}+R_{n-1, b}\right) R_{n}+\left(R_{n, a}+R_{n, b}\right) R_{n-1}+R_{n-1, a} R_{n, b}+R_{n-1, b} R_{n, a}\right) \\
& \quad-2 r_{n}\left(r_{n, a}+r_{n, b}\right)+(\alpha-a) r_{n, a}+(\alpha-b) r_{n, b}-2 r_{n, a} r_{n, b} .
\end{aligned}
$$

Now, we use (4.9) and (4.23) to eliminate $r_{n}$ and $R_{n}\left(R_{n-1}\right)$, (4.14) and (4.15) to eliminate $R_{n-1, a}$ and $R_{n-1, b}$ in the above equation, it follows that

$$
\begin{aligned}
a \sum_{j=0}^{n-1} R_{j, a}+b \sum_{j=0}^{n-1} R_{j, b}= & \beta_{n}\left(R_{n, a}+R_{n, b}\right)+\frac{r_{n, a}^{2}}{R_{n, a}}\left(1-R_{n, b}\right)+\frac{r_{n, b}^{2}}{R_{n, b}}\left(1-R_{n, a}\right) \\
& +(2 n+\alpha-a) r_{n, a}+(2 n+\alpha-b) r_{n, b}+2 r_{n, a} r_{n, b} .
\end{aligned}
$$

\subsection{Toda evolution}

As in the GUE case, it is easily verified that, in the present case, we still have

$$
\partial_{a} \ln h_{n}=-R_{n, a}, \quad \partial_{b} \ln h_{n}=-R_{n, b} .
$$




$$
\partial_{a} \ln \beta_{n}=R_{n-1, a}-R_{n, a}, \quad \partial_{b} \ln \beta_{n}=R_{n-1, b}-R_{n, b},
$$

with $R_{n, a}$ and $R_{n, b}$ defined in (4.4) and (4.5), respectively, and

$$
\begin{aligned}
& \partial_{a} \mathrm{p}_{1}(n, a, b)=r_{n, a}, \quad \partial_{b} \mathrm{p}_{1}(n, a, b)=r_{n, b} . \\
& \partial_{a} \alpha_{n}=r_{n, a}-r_{n+1, a}, \quad \partial_{b} \alpha_{n}=r_{n, b}-r_{n+1, b},
\end{aligned}
$$

where $r_{n, a}$ and $r_{n, b}$ are given in (4.7) and (4.8), respectively.

With the above preparations, we are ready to state a lemma which gives a two variable version of the Toda equation for the recurrence coefficients $\alpha_{n}$ and $\beta_{n}$ in the present case:

Lemma 5. For LUE, we have

$$
\begin{aligned}
\left(a \partial_{a}+b \partial_{b}\right) \beta_{n} & =\beta_{n}\left(\alpha_{n-1}-\alpha_{n}+2\right), \\
\left(a \partial_{a}+b \partial_{b}-1\right) \alpha_{n} & =\beta_{n}-\beta_{n+1} .
\end{aligned}
$$

Proof. From (4.28), it is easily seen that

$$
\frac{\left(a \partial_{a}+b \partial_{b}\right) \beta_{n}}{\beta_{n}}=a R_{n-1, a}+b R_{n-1, b}-\left(a R_{n, a}+b R_{n, b}\right) .
$$

Taking into account of (4.24), this gives

$$
\frac{\left(a \partial_{a}+b \partial_{b}\right) \beta_{n}}{\beta_{n}}=\alpha_{n-1}-\alpha_{n}+2,
$$

which is (4.31).

To establish (4.32), we make the following decomposition

$$
\left(a \partial_{a}+b \partial_{b}\right) \alpha_{n}=\left(\left(a-\alpha_{n}\right) \partial_{a}+\left(b-\alpha_{n}\right) \partial_{b}\right) \alpha_{n}+\alpha_{n}\left(\partial_{a}+\partial_{b}\right) \alpha_{n} .
$$

By (4.30) and (4.23), we have

$$
\alpha_{n}\left(\partial_{a}+\partial_{b}\right) \alpha_{n}=\alpha_{n}\left(r_{n, a}+r_{n, b}-r_{n+1, a}-r_{n+1, b}\right)=\alpha_{n}\left(1+r_{n+1}-r_{n}\right) .
$$

On the other hand, it follows from (4.30), (4.21), (4.22) and (4.9) that

$$
\begin{aligned}
& \left(\left(a-\alpha_{n}\right) \partial_{a}+\left(b-\alpha_{n}\right) \partial_{b}\right) \alpha_{n} \\
& =\left(a-\alpha_{n}\right)\left(r_{n, a}-r_{n+1, a}\right)+\left(b-\alpha_{n}\right)\left(r_{n, b}-r_{n+1, b}\right) \\
& =\beta_{n}\left(R_{n-1, a}+R_{n-1, b}\right)-\beta_{n+1}\left(R_{n+1, a}+R_{n+1, b}\right) \\
& =\beta_{n}-\beta_{n+1}+\beta_{n+1} R_{n+1}-\beta_{n} R_{n-1} \\
& =\beta_{n}-\beta_{n+1}+\alpha_{n}\left(r_{n}-r_{n+1}\right),
\end{aligned}
$$

where we also make use of (4.20) in the last step of the above equation. Substituting (4.37) and (4.36) into (4.35) gives us (4.32). 


\subsection{Derivation of partial differential equation}

We set

$$
H_{n}(a, b):=\left(a \partial_{a}+b \partial_{b}\right) \ln D_{n}(a, b),
$$

where $D_{n}$ is the Hankel determinant defined in (2.5). It is the aim of this section to derive a pde satisfied by $H_{n}$.

We note that, the equations (3.26) and (3.27) still hold in the present case, it then follows from (4.26) that

$$
\begin{aligned}
H_{n}= & -a \sum_{j=0}^{n-1} R_{j, a}-b \sum_{j=0}^{n-1} R_{j, b} \\
= & (a-\alpha-2 n) r_{n, a}+(b-\alpha-2 n) r_{n, b}-2 r_{n, a} r_{n, b} \\
& -\beta_{n}\left(R_{n, a}+R_{n, b}\right)-\frac{r_{n, a}^{2}}{R_{n, a}}\left(1-R_{n, b}\right)-\frac{r_{n, b}^{2}}{R_{n, b}}\left(1-R_{n, a}\right) .
\end{aligned}
$$

In view of (4.24) and (2.11), we also have

$$
\begin{aligned}
H_{n} & =-a \sum_{j=0}^{n-1} R_{j, a}-b \sum_{j=0}^{n-1} R_{j, b} \\
& =\sum_{j=0}^{n-1}\left(\alpha+2 j+1-\alpha_{j}(a, b)\right)=n(\alpha+n)+\mathrm{p}_{1}(n, a, b) .
\end{aligned}
$$

This, together with (4.29), implies

$$
\partial_{a} H_{n}=r_{n, a}, \quad \partial_{b} H_{n}=r_{n, b} .
$$

Next, we derive representations of $\beta_{n}, R_{n, a}$ and $R_{n, b}$ in terms of $H_{n}$ and its partial derivatives. To this end, we use (4.23) and (4.9) to eliminate $r_{n}$ and $R_{n}$ in (4.13), and then use (4.14) and (4.15) to eliminate the resulting $R_{n-1, a}$ and $R_{n-1, b}$, it follows that

$$
\begin{aligned}
& -\beta_{n}\left(R_{n, a}+R_{n, b}\right)-\frac{r_{n, a}^{2}}{R_{n, a}}\left(1-R_{n, b}\right)-\frac{r_{n, b}^{2}}{R_{n, b}}\left(1-R_{n, a}\right) \\
& =-\beta_{n}+\left(n+r_{n, a}+r_{n, b}\right)^{2}+\alpha\left(n+r_{n, a}+r_{n, b}\right)-r_{n, a}^{2}-r_{n, b}^{2} .
\end{aligned}
$$

Inserting the above equation into (4.39), we obtain after some simplification that

$$
\beta_{n}=n(n+\alpha)-H_{n}+a r_{n, a}+b r_{n, b},
$$


or equivalently, taking into account of (4.41),

$$
\beta_{n}=n^{2}+\alpha n-H_{n}+a \partial_{a} H_{n}+b \partial_{b} H_{n} .
$$

From (4.28), (4.14) and (4.15), we further have

$$
\partial_{a} \beta_{n}=\frac{r_{n, a}^{2}}{R_{n, a}}-\beta_{n} R_{n, a}, \quad \partial_{b} \beta_{n}=\frac{r_{n, b}^{2}}{R_{n, b}}-\beta_{n} R_{n, b}
$$

Using (4.44) and (4.41) in (4.45), it is readily seen that

$$
\begin{aligned}
& a \partial_{a}^{2} H_{n}+b \partial_{a} \partial_{b} H_{n}=\frac{\left(\partial_{a} H_{n}\right)^{2}}{R_{n, a}}-\left(n^{2}+\alpha n-H_{n}+a \partial_{a} H_{n}+b \partial_{b} H_{n}\right) R_{n, a}, \\
& b \partial_{b}^{2} H_{n}+a \partial_{a} \partial_{b} H_{n}=\frac{\left(\partial_{b} H_{n}\right)^{2}}{R_{n, b}}-\left(n^{2}+\alpha n-H_{n}+a \partial_{a} H_{n}+b \partial_{b} H_{n}\right) R_{n, b} .
\end{aligned}
$$

Solving the above quadratic equations for $R_{n, a}$ and $R_{n, b}$, we obtain

$$
\begin{aligned}
R_{n, a} & =\frac{-\left(a \partial_{a}^{2} H_{n}+b \partial_{a} \partial_{b} H_{n}\right) \pm \sqrt{\Delta_{1}}}{2\left(n^{2}+\alpha n-H_{n}+a \partial_{a} H_{n}+b \partial_{b} H_{n}\right)}, \\
R_{n, b} & =\frac{-\left(b \partial_{b}^{2} H_{n}+a \partial_{a} \partial_{b} H_{n}\right) \pm \sqrt{\Delta_{2}}}{2\left(n^{2}+\alpha n-H_{n}+a \partial_{a} H_{n}+b \partial_{b} H_{n}\right)},
\end{aligned}
$$

where

$$
\Delta_{1}=\left(a \partial_{a}^{2} H_{n}+b \partial_{a} \partial_{b} H_{n}\right)^{2}+4\left(\partial_{a} H_{n}\right)^{2}\left(n^{2}+\alpha n-H_{n}+a \partial_{a} H_{n}+b \partial_{b} H_{n}\right)
$$

and

$$
\Delta_{2}=\left(b \partial_{b}^{2} H_{n}+a \partial_{a} \partial_{b} H_{n}\right)^{2}+4\left(\partial_{b} H_{n}\right)^{2}\left(n^{2}+\alpha n-H_{n}+a \partial_{a} H_{n}+b \partial_{b} H_{n}\right) .
$$

Finally, substituting (4.41), (4.44), (4.48) and (4.49) into (4.39) yields

$$
\begin{aligned}
& 2\left(n^{2}+\alpha n-H_{n}+a \partial_{a} H_{n}+b \partial_{b} H_{n}\right)\left(H_{n}+(2 n+\alpha-a) \partial_{a} H_{n}+(2 n+\alpha-b) \partial_{b} H_{n}+2 \partial_{a} H_{n} \partial_{b} H_{n}\right) \\
& +\left(a \partial_{a}^{2} H_{n}+b \partial_{a} \partial_{b} H_{n}\right)\left(b \partial_{b}^{2} H_{n}+a \partial_{a} \partial_{b} H_{n}\right) \\
& =2\left(n^{2}+\alpha n-H_{n}+a \partial_{a} H_{n}+b \partial_{b} H_{n}\right)\left( \pm \sqrt{\Delta_{1}} \pm \sqrt{\Delta_{2}}\right) \pm \sqrt{\Delta_{1} \Delta_{2}}
\end{aligned}
$$

where $\Delta_{i}, i=1,2$ is given in (4.50) and (4.51), respectively. Denote by

$$
\begin{aligned}
k:= & 2\left(n^{2}+\alpha n-H_{n}+a \partial_{a} H_{n}+b \partial_{b} H_{n}\right)\left(H_{n}+(2 n+\alpha-a) \partial_{a} H_{n}+(2 n+\alpha-b) \partial_{b} H_{n}\right. \\
& \left.+2 \partial_{a} H_{n} \partial_{b} H_{n}\right)+\left(a \partial_{a}^{2} H_{n}+b \partial_{a} \partial_{b} H_{n}\right)\left(b \partial_{b}^{2} H_{n}+a \partial_{a} \partial_{b} H_{n}\right)
\end{aligned}
$$

and

$$
l:=2\left(n^{2}+\alpha n-H_{n}+a \partial_{a} H_{n}+b \partial_{b} H_{n}\right),
$$


we can rewrite equation (4.52) in the following equivalent form:

$$
\begin{aligned}
& \left(\left(\left(k^{2}-l^{2}\left(\Delta_{1}+\Delta_{2}\right)-\Delta_{1} \Delta_{2}\right)^{2}-4 l^{2} \Delta_{1} \Delta_{2}\left(l^{2}+\sqrt{\Delta_{1} \Delta_{2}}+\Delta_{1}+\Delta_{2}\right)\right)^{2}\right. \\
& \left.-16 l^{6}\left(\Delta_{1} \Delta_{2}\right)^{2}\left(\Delta_{1}+\Delta_{2}\right)\right)^{2}-1024 l^{12}\left(\Delta_{1} \Delta_{2}\right)^{5}=0 .
\end{aligned}
$$

Suppose there is no $a$-dependence in $H_{n}$, the equation (4.53) reduces to

$$
\left(b \partial_{b}^{2} H_{n}\right)^{2}=\left(H_{n}+(2 n+\alpha-b) \partial_{b} H_{n}\right)^{2}-4\left(\partial_{b} H_{n}\right)^{2}\left(n^{2}+\alpha n-H_{n}+b \partial_{b} H_{n}\right) .
$$

The equation (4.54) is nothing but the Okamoto-Jimbo-Miwa $\sigma$-form of the Painlevé V equation [25]:

$$
\begin{aligned}
\left(z \sigma^{\prime \prime}\right)^{2}= & \left(\sigma-z \sigma^{\prime}+2\left(\sigma^{\prime}\right)^{2}+\left(\nu_{0}+\nu_{1}+\nu_{2}+\nu_{3}\right) \sigma^{\prime}\right)^{2} \\
& -4\left(\sigma^{\prime}+\nu_{0}\right)\left(\sigma^{\prime}+\nu_{1}\right)\left(\sigma^{\prime}+\nu_{2}\right)\left(\sigma^{\prime}+\nu_{3}\right)
\end{aligned}
$$

with

$$
\nu_{0}=n, \quad \nu_{1}=n+\alpha, \quad \nu_{2}=\nu_{3}=0 .
$$

We have the same conclusion if there is no $b$-independence in $H_{n}$.

\subsection{Scaling of PDE}

In this section, we will scale the pde obtained in (4.52) and show that its solution is asymptotically equal to the sum of Tracy-Widom left and right distributions under certain delicate scaling.

For this purpose, we set

$$
\alpha=\beta n, \quad \beta>0,
$$

and denote by

$$
L:=2+\beta-2 \sqrt{2+\beta}, \quad R:=2+\beta+2 \sqrt{2+\beta} .
$$

Note that $L R=\beta^{2}$. We then scale the left and right soft edges $a$ and $b$ as follows:

$$
a=L n+c L^{2 / 3} n^{1 / 3} x, \quad b=R n+c R^{2 / 3} n^{1 / 3} y,
$$

with $c>0$. Clearly, it is easily seen that

$$
\partial_{a}=\frac{\partial_{x}}{c L^{2 / 3} n^{1 / 3}}, \quad \partial_{b}=\frac{\partial_{y}}{c R^{2 / 3} n^{1 / 3}},
$$

and

$$
a \partial_{a}=\left(\frac{L^{1 / 3}}{c} n^{2 / 3}+x\right) \partial_{x}, \quad b \partial_{b}=\left(\frac{R^{1 / 3}}{c} n^{2 / 3}+y\right) \partial_{y}
$$


Recall

$$
H_{n}(a, b)=\left(a \partial_{a}+b \partial_{b}\right) \ln D_{n}(a, b)=\left(a \partial_{a}+b \partial_{b}\right) \ln \operatorname{Prob}(n, a, b),
$$

and in the new variables $x, y$ this becomes

$$
\begin{aligned}
& H_{n}\left(L n+c L^{2 / 3} n^{1 / 3} x, R n+c R^{2 / 3} n^{1 / 3} y\right) \\
& =\left[\left(\frac{L^{1 / 3}}{c} n^{2 / 3}+x\right) \partial_{x}+\left(\frac{R^{1 / 3}}{c} n^{2 / 3}+y\right) \partial_{y}\right] \ln D_{n} .
\end{aligned}
$$

Let

$$
\widetilde{H}(x, y, n):=\frac{1}{n^{2 / 3}} H_{n}\left(L n+c L^{2 / 3} n^{1 / 3} x, R n+c R^{2 / 3} n^{1 / 3} y\right) .
$$

After substituting the change of variables (4.59), the leading term of (4.52) is of order $n^{8 / 3}$ and produces the following limiting pde:

$$
\begin{aligned}
& 4 c^{3} \sqrt{1+\beta} \beta^{4 / 3} \partial_{x} \widetilde{H} \partial_{y} \widetilde{H}\left(\widetilde{H}-x \partial_{x} \widetilde{H}-y \partial_{y} \widetilde{H}\right) \\
& +\partial_{x} \widetilde{H}\left(L^{4 / 3}\left(\partial_{x} \partial_{y} \widetilde{H}\right)^{2}+\beta^{4 / 3}\left(\partial_{y}^{2} \widetilde{H}\right)^{2}+2 \beta^{2 / 3} L^{2 / 3} \partial_{x} \partial_{y} \widetilde{H} \partial_{y}^{2} \widetilde{H}+4 c \beta^{2 / 3} L^{1 / 3}\left(\partial_{y} \widetilde{H}\right)^{3}\right) \\
& -\partial_{y} \widetilde{H}\left(R^{4 / 3}\left(\partial_{x} \partial_{y} \widetilde{H}\right)^{2}+\beta^{4 / 3}\left(\partial_{x}^{2} \widetilde{H}\right)^{2}+2 \beta^{2 / 3} R^{2 / 3} \partial_{x} \partial_{y} \widetilde{H} \partial_{x}^{2} \widetilde{H}+4 c \beta^{2 / 3} R^{1 / 3}\left(\partial_{x} \widetilde{H}\right)^{3}\right)=0 .
\end{aligned}
$$

As in GUE case, it turns out that pde (4.65) admits the following factorization ansatz:

$$
\widetilde{H}(x, y)=f(x)+g(y),
$$

where $f$ and $g$ satisfy the $\sigma$-form of a particular Painlevé II, respectively. More precisely,

$$
\begin{aligned}
& \left(f^{\prime \prime}(x)\right)^{2}=4 c^{3} \sqrt{1+\beta} f(x) f^{\prime}(x)-4 c^{3} \sqrt{1+\beta} x\left(f^{\prime}(x)\right)^{2}-\frac{4 c}{L^{1 / 3}}\left(f^{\prime}(x)\right)^{3}, \\
& \left(g^{\prime \prime}(y)\right)^{2}=-4 c^{3} \sqrt{1+\beta} g(y) g^{\prime}(y)+4 c^{3} \sqrt{1+\beta} y\left(g^{\prime}(y)\right)^{2}-\frac{4 c}{R^{1 / 3}}\left(g^{\prime}(y)\right)^{3} .
\end{aligned}
$$

It is readily seen that (4.66)-(4.68) satisfy (4.65) identically. This proves that

$$
\lim _{n \rightarrow \infty} \frac{1}{n^{2 / 3}} H_{n}\left(L n+c L^{2 / 3} n^{1 / 3} x, R n+c R^{2 / 3} n^{1 / 3} y\right)=f(x)+g(y),
$$

where $f$ and $g$ is Tracy-Widom left and right distribution, respectively.

Acknowledgements We would like to thank Iain Johnstone for bringing this problem to our attention. Lun Zhang is supported in part by the Belgian Interuniversity Attraction Pole P06/02m and by FWO-Flanders project G.0427.09. 


\section{References}

[1] M. Adler and P. Van Moerbeke, Matrix integrals, Toda symmetries, Virasoro constraints, and orthogonal polynomials, Duke Math. J. 80 (1995) 863-891.

[2] M. Adler and P. Van Moerbeke, Hermitian, symmetric and symplectic random ensembles: PDEs for the distribution of the spectrum, Annal of Math. 153 (2001) 149-189.

[3] Random matrix theory and its applications: Multivariate statistics and wireless communications (Lecture Notes Series, Institute for Mathematical Sciences, National University of Singapore), Eds. Zhi Dong Bai, Yang Chen and Ying-chang Liang, World Scientific, 2009.

[4] E. Basor and Y. Chen, Toeplitz determinants from compatibility conditions, Ramanujan J. 16 (2008) 25-40.

[5] E. Basor, Y. Chen and T. Ehrhardt, Painlevé V and time-dependent Jacobi polynomials, J. Phys. A: Math. Theor. 43 (2010) 015204 (25 pp).

[6] P. Bianchi, M. Debbah and J. Najim, Asymtoptic independence in the spectrum of Gaussian Unitary Ensemble, arXiv:0811.0979v1.

[7] L. Boelen, Discrete Painlevé Equations and Orthogonal Polynomials, Ph. D. thesis, K. U. Leuven, 2010.

[8] L. Boelen, G. Filipuk and W. Van Assche, Recurrence coefficients of generalized Meixner polynomials and Painlevé equations, J. Phys. A: Math. Theor. 44 (2011) 035202 (19 pp).

[9] L. Boelen and W. Van Assche, Discrete Painlevé equations for recurrence coefficients of semiclassical Laguerre polynomials, Proc. Amer. Math. Soc. 138 (2010) 1317-1331.

[10] F. Bornemann, Asymptotic independence of the extreme eigenvalues Gaussian Unitary ensembles, J. Math. Phys. 51 (2010) 023514 (8 pp).

[11] Y. Chen and D. Dai, Painlevé V and a Pollaczek-Jacobi type polynomials, J. Approx. Theory, 162 (2010) 2149-2167.

[12] Y. Chen and M. V. Feigin, Painlevé IV and degenerate Gaussian unitary ensembles, J. Phys. A: Math. Gen. 39 (2006) 12381-12393.

[13] Y. Chen and M. E. H. Ismail, Ladder operators and differential equations for orthogonal polynomials, J. Phys. A: Math. Gen. 30 (1997) 7817-7829.

[14] Y. Chen and M. E. H. Ismail, Jacobi polynomials from compatibility conditios, Proc. Amer. Math. Soc. 133 (2005) 465-472. 
[15] Y. Chen and M. E. H. Ismail, Ladder operators for q-orthogonal polynomials, J. Math. Anal. Appl. 345 (2008) 1-10.

[16] Y. Chen and A. Its, Painlevé III and a singular linear statistics in Hermitian random matrix ensembles, I., J. Approx. Theory 162 (2010) 270-297.

[17] Y. Chen and G. Pruessner, Orthogonal polynomials with discontinuous weight, J. Phys. A: Math. Gen. 38 (2005) L191-L198.

[18] Y. Chen and M. R. Mckay, Perturbed Hankel determinants: Applications to the information theory of MIMO wireless communications, arXiv:1007.0496.

[19] Y. Chen and L. Zhang, Painlevé VI and the unitary Jacobi ensemble, Stud. Appl. Math. 125 (2010) 91-112.

[20] D. Dai and L. Zhang, Painlevé VI and Hankel determinants for the generalized Jacobi weight, J. Phys. A: Math. Theor. 43 (2010) 055207 (14 pp).

[21] P. Deift, Universality for mathematical and physical systems, International Congress of Mathematicians, Vol. I, Eur. Math. Soc., Zürich, (2007) 125-152.

[22] P. J. Forrester and C. M. Ormerod, Differential equations for deformed Laguerre polynomials, J. Approx. Theory 162 (2010) 653-677.

[23] M. E. H. Ismail and Z. S. I. Mansour, q-Analogues of Freud weights and nonlinear difference equations, Adv. in Appl. Math. 45 (2010) 518-547.

[24] M. E. H. Ismail, I. Nikonova and P. Simeonov, Difference equations and discriminants for discrete orthogonal polynomials, Ramanujan J. 8 (2004) 475-502.

[25] M. Jimbo and T. Miwa, Monodromy perserving deformation of linear ordinary differential equations with rational coefficients, II, Physica D 2 (1981) 407-448.

[26] M. Jimbo, T. Miwa and K. Ujimo, Monodromy preserving deformation of linear ordinary differential equations with rational coefficients. I. General theory and $\tau$-function, Physica D. 2 (1981) 306-352.

[27] M. L. Mehta, Random Matrices, Third Ed. Elsevier, New York, 2004.

[28] G. Szegő, Orthogonal poynomials, AMS Colloquium Publications, vol. 23, 4th Ed. Providence, RI, 1975.

[29] C. A. Tracy and H. Widom, Level-spacing distributions and the Airy kernel, Commun. Math. Phys. 159 (1994) 151-174.

[30] C. A. Tracy and H. Widom, Fredholm determinants, differential equations and matrix models, Commun. Math. Phys. 163 (1994) 33-72. 
[31] T. Tsuda, Tau function of the fourth Painlevé equation in two variables, Funkcial. Ekvac. 48 (2005) 137-145. 